\title{
THE WEAK METRIC APPROXIMATION PROPERTY
}

\author{
ÅSVALD LIMA AND EVE OJA
}

\begin{abstract}
We introduce and investigate the weak metric approximation property of Banach spaces which is strictly stronger than the approximation property and at least formally weaker than the metric approximation property. Among others, we show that if a Banach space has the approximation property and is 1-complemented in its bidual, then it has the weak metric approximation property. We also study the lifting of the weak metric approximation property from Banach spaces to their dual spaces. This enables us, in particular, to show that the subspace of $c_{0}$, constructed by Johnson and Schechtman, does not have the weak metric approximation property.
\end{abstract}

\section{INTRODUCTION}

Let $X$ and $Y$ be Banach spaces. We denote by $\mathcal{L}(X, Y)$ the Banach space of bounded linear operators from $X$ to $Y$, and by $\mathcal{F}(X, Y), \mathcal{K}(X, Y)$, and $\mathcal{W}(X, Y)$ its subspaces of finite rank operators, compact operators, and weakly compact operators.

Recall that a Banach space $X$ is said to have the approximation property (AP) if there exists a net $\left(S_{\alpha}\right) \subset \mathcal{F}(X, X)$ such that $S_{\alpha} \rightarrow I_{X}$ uniformly on compact subsets of $X$. If $\left(S_{\alpha}\right)$ can be chosen with $\sup _{\alpha}\left\|S_{\alpha}\right\| \leq 1$, then $X$ is said to have the metric approximation property (MAP).

The following is a famous open problem (see, e.g., [1, page 289]).

Problem 1.1. Does the approximation property of the dual space $X^{*}$ of a Banach space $X$ imply the metric approximation property of $X^{*}$ ?

In Section 2 of this paper, we introduce the weak metric approximation property of Banach spaces and make a preliminary study of it in a more general context of the weak $\lambda$-bounded approximation property. The weak metric approximation property is strictly stronger than the AP and at least formally weaker than the MAP. But, for example, it is different from the 8-bounded approximation property.

In Section 3, we describe the weak metric approximation property in terms of trace mappings. Among other things, we show that the approximation property of $X^{*}$ implies the weak metric approximation property of $X^{*}$. Thus Problem 1.1 is equivalent to the following.

Problem 1.2. Does the weak metric approximation property of $X^{*}$ imply the metric approximation property of $X^{*}$ ?

2000 Mathematics Subject Classification. 46B04, 46B20, 46B28, 46M05, 47L05.

Key words and phrases. Approximation properties, projective tensor product of Banach spaces, trace mappings.

The research of the second-named author was partially supported by Estonian Science Foundation Grant 5704 and the Norwegian Academy of Science and Letters. 
It is well known (see, e.g. [3, page 193]) that $X^{*}$ has the MAP if and only if, for every Banach space $Y$, the trace mapping $V: X^{*} \hat{\otimes}_{\pi} Y \rightarrow \mathcal{F}(Y, X)^{*}$ is isometric. Our results in Section 3 show that Problem 1.1 is equivalent to the following.

Problem 1.3. If, for every reflexive Banach space $Y$, the trace mapping $V$ : $X^{*} \hat{\otimes}_{\pi} Y \rightarrow \mathcal{F}(Y, X)^{*}$ is isometric, must $X^{*}$ have the metric approximation property?

In Section 4, we study the lifting of the weak metric approximation property from Banach spaces to their dual spaces. This enables us, in particular, to show that the subspace of $c_{0}$, constructed by Johnson and Schechtman, does not have the weak metric approximation property. This also permits us to reformulate Problem 1.1 as follows.

Problem 1.4. If $X$ has the weak metric approximation property in every equivalent norm, must $X^{*}$ have the metric approximation property?

The main tool that we shall use in our proofs is the isometric version of the famous Davis-Figiel-Johnson-Pełczyński factorization lemma [2] due to Lima, Nygaard, and Oja [13]. In this paper, it will simply be called the factorization lemma. We shall apply it in several different situations and, in summa summarum, we probably need all aspects of it.

Let us fix some more notation. We consider normed linear spaces (Banach spaces) over the same field of real or complex numbers. In a linear normed space $X$, we denote $B_{X}(0, r)=\{x \in X:\|x\| \leq r\}$ and $B_{X}=B_{X}(0,1)$. The closure of a set $A \subset X$ is denoted by $\bar{A}$, its linear span by span $A$, its convex hull by conv $A$, and its absolutely convex hull by absconv $A$. We shall always regard $X$ as a subspace of $X^{* *}$. Thus the identity operator $I_{X}$ on $X$ is also considered as the embedding, identifying $I_{X}$ with the canonical embedding $j_{X}: X \rightarrow X^{* *}$. We denote the set of all weak* strongly exposed points of $B_{X^{*}}$ by $w^{*}$-sexp $B_{X^{*}}$.

\section{THE WEAK BOUNDED APPROXIMATION PROPERTY}

Let $X$ be a Banach space and let $1 \leq \lambda<\infty$. Let us recall that $X$ is said to have the $\lambda$-bounded approximation property ( $\lambda$-BAP) if there exists a net $\left(S_{\alpha}\right) \subset \mathcal{F}(X, X)$ with $\sup _{\alpha}\left\|S_{\alpha}\right\| \leq \lambda$ such that $S_{\alpha} \rightarrow I_{X}$ uniformly on compact subsets of $X$.

We shall say that $X$ has the weak $\lambda$-bounded approximation property (weak $\lambda$-BAP) if for every Banach space $Y$ and for every operator $T \in \mathcal{W}(X, Y)$, there exists a net $\left(S_{\alpha}\right) \subset \mathcal{F}(X, X)$ with $\sup _{\alpha}\left\|T S_{\alpha}\right\| \leq \lambda\|T\|$ such that $S_{\alpha} \rightarrow I_{X}$ uniformly on compact subsets of $X$. We say that $X$ has the weak bounded approximation property (weak BAP) if $X$ has the weak $\lambda$-BAP for some $\lambda$. And we say that $X$ has the weak metric approximation property (weak MAP) if $X$ has the weak 1-BAP.

Let us start with the following observations.

Proposition 2.1. If $X$ has the $\lambda-B A P$, then $X$ has the weak $\lambda$-BAP. If $X$ has the weak BAP, then $X$ has the AP.

Proof. The first assertion is immediate from the definitions. So is the second one if we take $T=0$. 
Proposition 2.2. There exists a separable Banach space $X$ such that all its duals are separable, $X$ has the AP, but $X$ does not have the weak BAP.

Proof. Let $X$ be the space of Reinov in [19]. Then $X, X^{*}, X^{* *}, \ldots$ are all separable, $X$ has the AP, and there exist a separable reflexive Banach space $Y$ and an operator $T \in \mathcal{K}(X, Y)$ such that for any $\lambda \geq 1$ the operator $T$ cannot be uniformly approximated on compact subsets of $X$ by finite rank operators with norms bounded by $\lambda$. This clearly contradicts the weak BAP.

We do not know whether the weak MAP and the MAP are different properties. We conjecture that they are. But, as the next result shows, the weak MAP and the BAP are in general different. And, for example, the weak MAP is different from the weak 8-BAP.

Proposition 2.3. There exists a closed subspace $X$ of $c_{0}$ such that $X$ has the 8-BAP, but $X$ does not have the weak MAP.

The space $X$ in Proposition 2.3 is the Johnson-Schechtman space (see [11, Corollary JS]). The proof of Proposition 2.3 relies on a lifting result from Section 4 (see Theorem 4.1) and will be given in Section 4.

Theorem 2.4. Let $X$ be a Banach space and let $1 \leq \lambda<\infty$. The following statements are equivalent.

(a) $X$ has the weak $\lambda$-BAP.

(b) For every separable reflexive Banach space $Y$ and for every operator $T \in \mathcal{K}(X, Y)$, there exists a net $\left(S_{\alpha}\right) \subset \mathcal{F}(X, X)$ with $\sup _{\alpha}\left\|T S_{\alpha}\right\| \leq$ $\lambda\|T\|$ such that $S_{\alpha} \rightarrow I_{X}$ in the strong operator topology.

(c) For every separable reflexive Banach space $Y$ and for every operator $T \in \mathcal{K}(X, Y)$, there exists a net $\left(S_{\alpha}\right) \subset \mathcal{F}(X, X)$ with $\sup _{\alpha}\left\|T S_{\alpha}\right\| \leq$ $\lambda\|T\|$ such that $T S_{\alpha} \rightarrow T$ in the strong operator topology.

(d) For every Banach space $Y$, for every operator $T \in \mathcal{W}(X, Y)$ with $\|T\|=1$, and for all sequences $\left(x_{n}\right) \subset X$, and $\left(y_{n}^{*}\right) \subset Y^{*}$ with $\sum_{n=1}^{\infty}\left\|x_{n}\right\|\left\|y_{n}^{*}\right\|<\infty$, one has the inequality

$$
\left|\sum_{n=1}^{\infty} y_{n}^{*}\left(T x_{n}\right)\right| \leq \lambda \sup _{\substack{\|T S\| \leq 1 \\ S \in \mathcal{F}(X, X)}}\left|\sum_{n=1}^{\infty} y_{n}^{*}\left(T S x_{n}\right)\right| .
$$

Proof. For the proof, it is convenient to introduce the following statements.

$\left(\mathrm{a}^{\prime}\right)$ The particular case of (a) with separable reflexive $Y$ and $T \in \mathcal{K}(X, Y)$.

$\left(\mathrm{d}^{\prime}\right)$ The particular case of (d) with separable reflexive $Y$ and $T \in \mathcal{K}(X, Y)$.

$\left(\mathrm{a}^{\prime}\right) \Rightarrow(\mathrm{b}) \Rightarrow(\mathrm{c})$ and $(\mathrm{d}) \Rightarrow\left(\mathrm{d}^{\prime}\right)$ are trivial.

$(\mathrm{c}) \Rightarrow\left(\mathrm{d}^{\prime}\right)$ and $(\mathrm{a}) \Rightarrow(\mathrm{d})$. Let $Y, T$ with $\|T\|=1,\left(x_{n}\right)$, and $\left(y_{n}^{*}\right)$ be as in $\left(\mathrm{d}^{\prime}\right)$ or $(\mathrm{d})$. Let $\varepsilon>0$ and choose $N \in \mathbb{N}$ such that $\sum_{n>N}\left\|x_{n}\right\|\left\|y_{n}^{*}\right\|<\varepsilon$. Choose $S \in \mathcal{F}(X, X)$ such that $\|T S\| \leq \lambda$ and $\left\|y_{n}^{*}\right\|\left\|T S x_{n}-T x_{n}\right\|<\varepsilon / N$ 
for all $n \leq N$. Then

$$
\begin{aligned}
\left|\sum_{n=1}^{\infty} y_{n}^{*}\left(T x_{n}\right)\right| & \leq\left|\sum_{n=1}^{N} y_{n}^{*}\left(T S x_{n}\right)\right|+\left|\sum_{n=1}^{N} y_{n}^{*}\left((T-T S) x_{n}\right)\right|+\sum_{n>N}\left\|x_{n}\right\|\|T\|\left\|y_{n}^{*}\right\| \\
& \leq\left|\sum_{n=1}^{\infty} y_{n}^{*}\left(T S x_{n}\right)\right|+(2+\lambda) \varepsilon .
\end{aligned}
$$

From this we get

$$
\left|\sum_{n=1}^{\infty} y_{n}^{*}\left(T x_{n}\right)\right| \leq \sup _{\substack{\|T S\| \leq \lambda \\ S \in \mathcal{F}(X, X)}}\left|\sum_{n=1}^{\infty} y_{n}^{*}\left(T S x_{n}\right)\right|=\lambda \sup _{\substack{\|T S\| \leq 1 \\ S \in \mathcal{F}(X, X)}}\left|\sum_{n=1}^{\infty} y_{n}^{*}\left(T S x_{n}\right)\right| .
$$

$\left(\mathrm{d}^{\prime}\right) \Rightarrow\left(\mathrm{a}^{\prime}\right)$. Let $Y$ and $T$ be as in $\left(\mathrm{a}^{\prime}\right)$. We may assume that $\|T\|=1$. We need to show that $I_{X}$ belongs to the closure of the absolutely convex set $\{S \in \mathcal{F}(X, X):\|T S\| \leq \lambda\} \subset \mathcal{L}(X, X)$ in the topology $\tau$ of uniform convergence on compact subsets of $X$. If this is not the case, then there exists $\varphi \in(\mathcal{L}(X, X), \tau)^{*}$ such that

$$
\left|\varphi\left(I_{X}\right)\right|>\sup \{|\varphi(S)|: S \in \mathcal{F}(X, X),\|T S\| \leq \lambda\} .
$$

By the description of $(\mathcal{L}(X, X), \tau)^{*}$, due to Grothendieck [8] (see, i.e., [14, page 31]), there exist $\left(x_{n}\right) \subset X$ and $\left(x_{n}^{*}\right) \subset X^{*}$ such that $\sum_{n}\left\|x_{n}^{*}\right\|\left\|x_{n}\right\|<$ $\infty$ and $\varphi(A)=\sum_{n} x_{n}^{*}\left(A x_{n}\right)$ for all $A \in \mathcal{L}(X, X)$. We may assume that $\sum_{n}\left\|x_{n}\right\|<\infty$ and $1 \geq\left\|x_{n}^{*}\right\| \rightarrow 0$.

Using the description of $\varphi$, we have

$$
\left|\sum_{n=1}^{\infty} x_{n}^{*}\left(x_{n}\right)\right|>\sup _{\substack{\|T S\| \leq \lambda \\ S \in \mathcal{F}(X, X)}}\left|\sum_{n=1}^{\infty} x_{n}^{*}\left(S x_{n}\right)\right|=\lambda \sup _{\substack{\|T S\| \leq 1 \\ S \in \mathcal{F}(X, X)}}\left|\sum_{n=1}^{\infty} x_{n}^{*}\left(S x_{n}\right)\right| .
$$

To show that this inequality cannot hold, we use the factorization lemma. Let $K=\overline{\operatorname{absconv}}\left(\left(x_{n}^{*}\right)_{n=1}^{\infty} \cup T^{*}\left(B_{Y^{*}}\right)\right) \subset B_{X^{*}}$. Since $K$ is compact, there exists a separable reflexive Banach space $Z$, which is a linear subspace of $X^{*}$, such that the identity embedding $J \in \mathcal{K}\left(Z, X^{*}\right),\|J\| \leq 1$, and $K \subset J\left(B_{Z}\right)$. Let $z_{n} \in B_{Z}$ be such that $x_{n}^{*}=J z_{n}$ for all $n$. Then $\sum_{n}\left\|x_{n}\right\|\left\|z_{n}\right\|<\infty$, and considering $\left.J^{*}\right|_{X} \in \mathcal{K}\left(X, Z^{*}\right)$ we have, by $\left(\mathrm{d}^{\prime}\right)$,

$$
\begin{aligned}
\left|\sum_{n=1}^{\infty} x_{n}^{*}\left(x_{n}\right)\right| & =\left|\sum_{n=1}^{\infty} z_{n}\left(J^{*} x_{n}\right)\right| \leq \lambda \sup _{\substack{\left\|J^{*} \mid X S\right\| \leq 1 \\
S \in \mathcal{F}(X, X)}}\left|\sum_{n=1}^{\infty} z_{n}\left(J^{*} S x_{n}\right)\right| \\
& \leq \lambda \sup _{\substack{\|T S\| \leq 1 \\
S \in \mathcal{F}(X, X)}}\left|\sum_{n=1}^{\infty} x_{n}^{*}\left(S x_{n}\right)\right|,
\end{aligned}
$$

because $\|T S\|=\left\|S^{*} T^{*}\right\| \leq\left\|S^{*} J\right\|=\left\|\left.J^{*}\right|_{X} S\right\|$ (recall that $T^{*}\left(B_{Y^{*}}\right) \subset$ $\left.J\left(B_{Z}\right)\right)$. The contradiction proves $\left(\mathrm{a}^{\prime}\right)$.

$\left(\mathrm{a}^{\prime}\right) \Rightarrow(\mathrm{a})$. Let $Y$ and $T$ be as in (a). We may assume that $\|T\|=1$. We also may assume that $Y$ is reflexive, because $T$ can be isometrically factorized through a reflexive Banach space (see [13, Theorem 2.2]). As in the previous proof, we need to show that $I_{X}$ belongs to the $\tau$-closure of $\{S \in \mathcal{F}(X, X):\|T S\| \leq \lambda\} \subset \mathcal{L}(X, X)$. 
Let $C$ be a compact subset of $X$ and let $\varepsilon>0$. Define

$$
\mathcal{C}=\{T S: S \in \mathcal{F}(X, X),\|S a-a\|<\varepsilon, a \in C\} \subset \mathcal{F}(X, Y) .
$$

We shall show that $\mathcal{C}$ cannot be strongly separated from $B_{\mathcal{F}(X, Y)}(0, \lambda)$. From this it then follows that, for all $\delta>0$, there is some $S \in \mathcal{F}(X, X)$ such that $T S \in \mathcal{C} \cap B_{\mathcal{F}(X, Y)}(0, \lambda+\delta)$. From this (a) follows.

Let $\varphi \in \mathcal{F}(X, Y)^{*}$ with $\|\varphi\|=1$. From a theorem of Feder and Saphar [5], for any $\eta>0$, we can find a representation of $\varphi$ as $\varphi=\sum_{n} y_{n}^{*} \otimes x_{n}^{* *} \in$ $Y^{*} \hat{\otimes}_{\pi} X^{* *}$ with $\sum_{n}\left\|y_{n}^{*}\right\|\left\|x_{n}^{* *}\right\|<1+\eta$. Moreover, we may assume that $1 \geq\left\|y_{n}^{*}\right\| \rightarrow 0$ and $\sum_{n}\left\|x_{n}^{* *}\right\|<1+2 \eta$. Let $K=\overline{\operatorname{absconv}}\left(\left(T^{*} y_{n}^{*}\right)_{n=1}^{\infty}\right) \subset B_{X^{*}}$. By the factorization lemma, since $K$ is compact, we can construct a separable reflexive Banach space $Z$, sitting inside $X^{*}$, such that the embedding operator $J \in \mathcal{K}\left(Z, X^{*}\right),\|J\| \leq 1$, and $K \subset J\left(B_{Z}\right)$. Let $z_{n} \in B_{Z}$ be such that $J z_{n}=T^{*} y_{n}^{*}$ for all $n$. We have $\left.J^{*}\right|_{X} \in \mathcal{K}\left(X, Z^{*}\right)$. Using ( $\left.\mathrm{a}^{\prime}\right)$, we can find $S \in \mathcal{F}(X, X)$ such that $\left\|\left.J^{*}\right|_{X} S\right\| \leq \lambda$ and $\|S a-a\|<\varepsilon$ for all $a \in C$. We get $\left\|J^{*} S^{* *}\right\| \leq \lambda$ and

$$
\begin{aligned}
|\varphi(T S)| & =\left|\sum_{n=1}^{\infty}\left(S^{* *} x_{n}^{* *}\right)\left(T^{*} y_{n}^{*}\right)\right|=\left|\sum_{n=1}^{\infty}\left(J^{*} S^{* *} x_{n}^{* *}\right)\left(z_{n}\right)\right| \\
& \leq \lambda \sum_{n=1}^{\infty}\left\|x_{n}^{* *}\right\|<\lambda(1+2 \eta) .
\end{aligned}
$$

This shows that

$$
\inf \{|\varphi(T S)|: T S \in \mathcal{C}\} \leq \lambda,
$$

so that $\mathcal{C}$ cannot be strongly separated from $B_{\mathcal{F}(X, Y)}(0, \lambda)$.

\section{The WEAK BOUNDED APPROXIMATION PROPERTY AND TRACE MAPPINGS}

Let us consider the trace mapping $V$ from the projective tensor product $X \hat{\otimes}_{\pi} Y^{*}$ to $\mathcal{F}(X, Y)^{*}$, the dual space of $\mathcal{F}(X, Y)$, defined by

$$
(V u)(T)=\operatorname{trace}(T u), \quad u \in X \hat{\otimes}_{\pi} Y^{*}, T \in \mathcal{F}(X, Y),
$$

that is, if $u=\sum_{n} x_{n} \otimes y_{n}^{*}$ with $\sum_{n}\left\|x_{n}\right\|\left\|y_{n}^{*}\right\|<\infty$, then

$$
(V u)(T)=\sum_{n} y_{n}^{*}\left(T x_{n}\right) .
$$

It is well known and easy to see that

$$
\|V u\| \leq\|u\|_{\pi}, \quad u \in X \hat{\otimes}_{\pi} Y^{*} .
$$

The following characterization of the $\lambda$-BAP, which is essentially due to Grothendieck [8], is well known.

Theorem 3.1 (see, e.g., [3, page 193]). A Banach space $X$ has the $\lambda$-BAP if and only if, for every Banach space $Y$, the trace mapping $V: X \hat{\otimes}_{\pi} Y^{*} \rightarrow$ $\mathcal{F}(X, Y)^{*}$ satisfies $\|u\|_{\pi} \leq \lambda\|V u\|$ for all $u \in X \hat{\otimes}_{\pi} Y^{*}$.

Defant and Floret [3, page 283] state as an open question whether it is enough, for the $\lambda$-BAP of $X$, to check the condition of Theorem 3.1 only for reflexive Banach spaces $Y$ (they conjecture that it is not). As the next 
result shows, this would precisely mean the equivalence of the $\lambda$-BAP and the weak $\lambda$-BAP.

Theorem 3.2. A Banach space $X$ has the weak $\lambda-B A P$ if and only if, for every reflexive Banach space $Y$, the trace mapping $V: X \hat{\otimes}_{\pi} Y^{*} \rightarrow \mathcal{F}(X, Y)^{*}$ satisfies $\|u\|_{\pi} \leq \lambda\|V u\|$ for all $u \in X \hat{\otimes}_{\pi} Y^{*}$.

Proof. Let $X$ have the weak $\lambda$-BAP and let $Y$ be a reflexive Banach space. Consider any $u=\sum_{n} x_{n} \otimes y_{n}^{*} \in X \hat{\otimes}_{\pi} Y^{*}$ with $\sum_{n}\left\|x_{n}\right\|\left\|y_{n}^{*}\right\|<\infty$. Since $\left(X \hat{\otimes}_{\pi} Y^{*}\right)^{*}=\mathcal{L}\left(X, Y^{* *}\right)=\mathcal{W}(X, Y)$, there exists $T \in \mathcal{W}(X, Y)$ with $\|T\|=$ 1 such that

$$
\|u\|_{\pi}=\operatorname{trace}(T u)=\sum_{n=1}^{\infty} y_{n}^{*}\left(T x_{n}\right) .
$$

Hence, using condition (d) of Theorem 2.4, we have

$$
\|u\|_{\pi} \leq \lambda \sup _{\substack{\|T S\| \leq 1 \\ S \in \mathcal{F}(X, X)}}\left|\sum_{n=1}^{\infty} y_{n}^{*}\left(T S x_{n}\right)\right| \leq \lambda \sup _{\substack{\|S\| \leq 1 \\ S \in \mathcal{F}(X, Y)}}\left|\sum_{n=1}^{\infty} y_{n}^{*}\left(S x_{n}\right)\right|=\lambda\|V u\| .
$$

To prove the converse, we shall verify condition (d) of Theorem 2.4. Let $Y$ be a Banach space, let $T \in \mathcal{W}(X, Y),\|T\|=1$, and let sequences $\left(x_{n}\right) \subset X$ and $\left(y_{n}^{*}\right) \subset Y^{*}$ satisfy $\sum_{n}\left\|x_{n}\right\|\left\|y_{n}^{*}\right\|<\infty$. We may assume that $\left\|y_{n}^{*}\right\|=1$, $n \in \mathbb{N}$.

Let $K=\overline{T^{*}\left(B_{Y^{*}}\right)}$. Since $K$ is weakly compact absolutely convex subset of $B_{X^{*}}$, by the factorization lemma, there exists a reflexive Banach space $Z$, which is a linear subspace of $X^{*}$, such that the identity embedding $J$ : $Z \rightarrow X^{*}$ has norm $\leq 1$ and $\overline{J^{*}\left(X^{* *}\right)}=Z^{*}$. Moreover, $T=\left.U J^{*}\right|_{X}$ for some operator $U \in \mathcal{L}\left(Z^{*}, Y\right)$ with $\|U\|=\|T\|=1$ and $T^{*}\left(B_{Y^{*}}\right) \subset J\left(B_{Z}\right)$. Let $z_{n} \in B_{Z}$ be such that $T^{*} y_{n}^{*}=J z_{n}, n \in \mathbb{N}$. Denote

$$
u=\sum_{n=1}^{\infty} x_{n} \otimes z_{n} \in X \hat{\otimes}_{\pi} Z
$$

and consider the trace mapping $V: X \hat{\otimes}_{\pi} Z \rightarrow \mathcal{F}\left(X, Z^{*}\right)^{*}$. By assumption, $\|u\|_{\pi} \leq \lambda\|V u\|$.

Before applying this inequality, let us show that

$$
B_{\mathcal{F}\left(X, Z^{*}\right)} \subset \overline{\left\{\left.J^{*}\right|_{X} S: S \in \mathcal{F}(X, X),\left\|\left.J^{*}\right|_{X} S\right\| \leq 1\right\}}
$$

in the norm topology of $\mathcal{F}\left(X, Z^{*}\right)$. First observe that $\overline{J^{*}(X)}=Z^{*}$. In fact, by the factorization lemma, we know that $\overline{J^{*}\left(X^{* *}\right)}=Z^{*}$. For any $x^{* *} \in X^{* *}$, there is a bounded net $\left(x_{\alpha}\right) \subset X$ such that $x_{\alpha} \rightarrow x^{* *}$ weak $^{*}$ in $X^{* *}$. Then $J^{*} x_{\alpha} \rightarrow J^{*} x^{* *}$ weak* in $Z^{*}$, hence weakly, because $Z^{*}$ is reflexive. Consequently, $J^{*} x^{* *} \in{\overline{J^{*}(X)}}^{w}=\overline{J^{*}(X)}$, and therefore $\overline{J^{*}(X)}=\overline{J^{*}\left(X^{* *}\right)}=$ $Z^{*}$. Finally, for any operator $\sum_{n=1}^{m} x_{n}^{*} \otimes z_{n}^{*} \in \mathcal{F}\left(X, Z^{*}\right)$, one can now find $u_{n} \in X$ with $\left\|z_{n}^{*}-J^{*} u_{n}\right\|$ so small that for $S=\sum_{n=1}^{m} x_{n}^{*} \otimes u_{n} \in \mathcal{F}(X, X)$, one has

$$
\left\|\sum_{n=1}^{m} x_{n}^{*} \otimes z_{n}^{*}-\left.J^{*}\right|_{X} S\right\|=\left\|\sum_{n=1}^{m} x_{n}^{*} \otimes\left(z_{n}^{*}-J^{*} u_{n}\right)\right\| \leq \sum_{n=1}^{m}\left\|x_{n}^{*}\right\|\left\|z_{n}^{*}-J^{*} u_{n}\right\|
$$

as small as one wants. From this, the above inclusion is clear. 
Now we can conclude our proof in an obvious way (recall that $\left.J^{*}\right|_{X} \in$ $\mathcal{L}\left(X, Z^{*}\right)=\left(X \hat{\otimes}_{\pi} Z\right)^{*}$ and $\|T S\| \leq\left\|\left.J^{*}\right|_{X} S\right\|$ for all $S \in \mathcal{F}(X, X)$ because $\left.T^{*}\left(B_{Y^{*}}\right) \subset J\left(B_{Z}\right)\right)$ :

$$
\begin{aligned}
\left|\sum_{n=1}^{\infty} y_{n}^{*}\left(T x_{n}\right)\right| & =\left|\sum_{n=1}^{\infty}\left(J^{*} x_{n}\right)\left(z_{n}\right)\right|=\left|\operatorname{trace}\left(J^{*} u\right)\right| \leq\|J\|\|u\|_{\pi} \\
& \leq\|u\|_{\pi} \leq \lambda\|V u\|=\lambda \sup _{S \in B_{\mathcal{F}\left(X, Z^{*}\right)}}|\operatorname{trace}(S u)| \\
& \leq \lambda \sup _{\substack{\left\|J^{*} \mid X S\right\| \leq 1 \\
S \in \mathcal{F}(X, X)}}\left|\operatorname{trace}\left(J^{*} S u\right)\right| \leq \lambda \sup _{\substack{\|T S\| \leq 1 \\
S \in \mathcal{F}(X, X)}}\left|\sum_{n=1}^{\infty}\left(J^{*} S x_{n}\right)\left(z_{n}\right)\right| \\
& =\lambda \sup _{\substack{\|T S\| \leq 1 \\
S \in \mathcal{F}(X, X)}}\left|\sum_{n=1}^{\infty} y_{n}^{*}\left(T S x_{n}\right)\right| .
\end{aligned}
$$

Corollary 3.3. Let a Banach space $X$ be complemented in its bidual $X^{* *}$ by a projection $P$. If $X$ has the $A P$, then $X$ has the weak $\|P\|-B A P$.

Proof. Let $Y$ be a reflexive Banach space. Consider the trace mapping $V: X \hat{\otimes}_{\pi} Y^{*} \rightarrow \mathcal{F}(X, Y)^{*}$ and let $u=\sum_{n} x_{n} \otimes y_{n}^{*} \in X \hat{\otimes}_{\pi} Y^{*}$. We need to show that $\|u\|_{\pi} \leq\|P\|\|V u\|$ (see Theorem 3.2). We are going to use some well-known facts from the theory of tensor products of Banach spaces (see, e.g., [4] or [20]).

Since $X$ has the $\operatorname{AP}, X \hat{\otimes}_{\pi} Y^{*}$ can be canonically identified with the Banach space $\mathcal{N}(Y, X)=\left(\mathcal{N}(Y, X),\|\cdot\|_{\mathcal{N}}\right)$ of nuclear operators. Let $U \in \mathcal{N}(Y, X)$ be canonically identified with $u$. Then $U y=\sum_{n} y_{n}^{*}(y) x_{n}, y \in Y$. On the other hand, we also have the canonical identification of $\mathcal{F}(X, Y)^{*}$ with the Banach space $\mathcal{I}\left(Y, X^{* *}\right)=\left(\mathcal{I}\left(Y, X^{* *}\right),\|\cdot\|_{\mathcal{I}}\right)$ of integral operators. The functional $V u$ considered as an integral operator acts as $(V u)(y)=\sum_{n} y_{n}^{*}(y) j_{X} x_{n}$. Now we clearly have $U=P \circ(V u)$. Since $V u \in \mathcal{N}\left(Y, X^{* *}\right)$ and $Y$ is reflexive, $\mathcal{N}\left(Y, X^{* *}\right)=\mathcal{I}\left(Y, X^{* *}\right)$ as Banach spaces. Consequently,

$$
\|u\|_{\pi}=\|U\|_{\mathcal{N}} \leq\|P\|\|V u\|_{\mathcal{N}}=\|P\|\|V u\|_{\mathcal{I}}=\|P\|\|V u\|
$$

as desired.

Corollary 3.4. Let $X$ be a Banach space. If $X^{*}$ has the AP, then $X^{*}$ has the weak MAP.

Proof. Use that $X^{*}$ is complemented in $X^{* * *}$ by $P=j_{X^{*}}\left(j_{X}\right)^{*}$ and apply Corollary 3.3.

Thus, for dual Banach spaces the AP and the weak MAP are equivalent properties. This means that Problems 1.1 and 1.2 (see Introduction) are equivalent.

For dual spaces, the well-known analogue of Theorem 3.1 reads as follows.

Theorem 3.5 (see, e.g., [3, page 193]). A dual space $X^{*}$ of a Banach space $X$ has the $\lambda$-BAP if and only if, for every Banach space $Y$, the trace mapping $V: X^{*} \hat{\otimes}_{\pi} Y \rightarrow \mathcal{F}(Y, X)^{*}$ satisfies $\|u\|_{\pi} \leq \lambda\|V u\|$ for all $u \in X^{*} \hat{\otimes}_{\pi} Y$. 
Using only reflexive Banach spaces instead of all Banach spaces gives the following.

Theorem 3.6. For a Banach space $X$, the following statements are equivalent.

(a) There exists $\lambda \geq 1$ such that, for every reflexive Banach space $Y$, the trace mapping $V: X^{*} \hat{\otimes}_{\pi} Y \rightarrow \mathcal{F}(Y, X)^{*}$ satisfies $\|u\|_{\pi} \leq \lambda\|V u\|$ for all $u \in X^{*} \hat{\otimes}_{\pi} Y$.

(b) For every reflexive Banach space $Y$, the trace mapping $V: X^{*} \hat{\otimes}_{\pi} Y \rightarrow$ $\mathcal{F}(Y, X)^{*}$ is isometric.

(c) $X^{*}$ has the weak MAP.

(d) $X^{*}$ has the $A P$.

Proof. (a) $\Rightarrow$ (d). Let us also consider the trace mapping $W: X^{*} \hat{\otimes}_{\pi} Y \rightarrow$ $\mathcal{F}\left(X^{*}, Y^{*}\right)^{*}$. Then, for all $u \in X^{*} \hat{\otimes}_{\pi} Y$,

$$
\begin{aligned}
\|u\|_{\pi} & \leq \lambda\|V u\|=\lambda \sup _{T \in B_{\mathcal{F}(Y, X)}}|\operatorname{trace}(T u)|=\lambda \sup _{T \in B_{\mathcal{F}(Y, X)}}\left|\operatorname{trace}\left(T^{*} u\right)\right| \\
& \leq \lambda \sup _{S \in B_{\mathcal{F}\left(X^{*}, Y^{*}\right)}}|\operatorname{trace}(S u)|=\lambda\|W u\| .
\end{aligned}
$$

Thus, by Theorem $3.2, X^{*}$ has the weak $\lambda$-BAP, hence $X^{*}$ has the AP.

(d) $\Rightarrow$ (c). See Corollary 3.4.

(c) $\Rightarrow$ (b). From Theorem 3.2, we know that the trace mapping $W$ above satisfies $\|u\|_{\pi}=\|W u\|$. To see that $\|W u\|=\|V u\|$, let us consider $W u$ and $V u$ as integral operators: $W u \in \mathcal{I}\left(Y^{*}, X^{* * *}\right)$ and $V u \in \mathcal{I}\left(Y^{*}, X^{*}\right)$. As in the proof of Corollary 3.3, one can easily verify that $W u=j_{X^{*}} \circ(V u)$. But then, by a well-known result of Grothendieck [8] (see, e.g., [4, page 233] or [20, page 65]), $\|W u\|_{\mathcal{I}}=\|V u\|_{\mathcal{I}}$ as desired.

(b) $\Rightarrow(\mathrm{a})$. This is obvious.

Remark 3.1. The trace mapping $V$ is surjective in Theorem 3.6 because the algebraic tensor product $X^{*} \otimes Y$ is dense in both spaces (recall that $\mathcal{F}(Y, X)^{*}=\mathcal{I}(X, Y)=\mathcal{N}(X, Y)$ since $Y$ is reflexive $)$.

Remark 3.2. Since $V$ is a bounded linear operator, one can clearly replace $X \hat{\otimes}_{\pi} Y^{*}$ by $X \otimes_{\pi} Y^{*}$ (the algebraic tensor product $X \otimes Y^{*}$ in its projective norm) in Theorems 3.1 and 3.2. And one can replace $X^{*} \hat{\otimes}_{\pi} Y$ by $X^{*} \otimes_{\pi} Y$ in Theorems 3.5 and 3.6.

In view of Theorem 3.6, one can reformulate Problem 1.1 as Problem 1.3 (see Introduction).

\section{LifTING THE WEAK METRIC APPROXIMATION PROPERTY FROM BANACH SPACES TO THEIR DUAL SPACES}

There are Banach spaces having special geometric structure that permits to lift the weak MAP from the space to its dual space. The following result applies, for example, to Banach spaces $X$ whose dual space $X^{*}$ has the Radon-Nikodým property and contains no proper norming closed subspace (see Remark 4.1 below).

Theorem 4.1. Let $X$ be a Banach space such that $X^{*}=\overline{\operatorname{span}}\left(w^{*}-\operatorname{sexp} B_{X^{*}}\right)$. If $X$ has the weak MAP, then $X^{*}$ has the weak MAP. 
Proof. Since the weak MAP and the AP are equivalent for $X^{*}$ (see Corollary 3.4), it suffices to prove that $X^{*}$ has the AP. This is equivalent to the following well-known condition (due to Grothendieck [8]; see, e.g., [14, Theorem 1.e.4]). For all sequences $\left(x_{n}^{*}\right) \subset X^{*}$ and $\left(x_{n}^{* *}\right) \subset X^{* *}$ such that $\sum_{n}\left\|x_{n}^{*}\right\|\left\|x_{n}^{* *}\right\|<\infty$ and $\sum_{n} x_{n}^{* *}\left(T x_{n}^{*}\right)=0$, whenever $T \in \mathcal{F}\left(X^{*}, X^{*}\right)$, one has $\sum_{n} x_{n}^{* *}\left(x_{n}^{*}\right)=0$.

Let $\left(x_{n}^{*}\right) \subset X^{*}$ and $\left(x_{n}^{* *}\right) \subset X^{* *}$ be as above. We may assume that $x_{n}^{*} \rightarrow 0, \sup _{n}\left\|x_{n}^{*}\right\| \leq 1$, and

$$
M:=\sum_{n=1}^{\infty}\left\|x_{n}^{* *}\right\|<\infty .
$$

We shall use the factorization lemma. Let

$$
c=\sqrt{\frac{1}{2}+\frac{2}{\ln a}}
$$

be the absolute constant, where $a>1$ is the unique solution of the equation

$$
\sum_{n=1}^{\infty} \frac{a^{n}}{\left(a^{n}+1\right)^{2}}=1
$$

We know that $c>1$.

For proving that $\sum_{n} x_{n}^{* *}\left(x_{n}^{*}\right)=0$, let us fix an arbitrary $\varepsilon>0$, assuming that $\varepsilon<4 M$. Choose $N \in \mathbb{N}$ such that

$$
\sum_{n>N}\left\|x_{n}^{* *}\right\|<\frac{\varepsilon}{8}
$$

Approximating $x_{1}^{*}, \ldots, x_{N}^{*}$ respectively by $\hat{x}_{1}^{*}, \ldots, \hat{x}_{N}^{*} \in B_{X^{*}} \cap \operatorname{span}\left(w^{*}-\operatorname{sexp} B_{X^{*}}\right)$ we have a finite subset $C$ of $w^{*}-\operatorname{sexp} B_{X^{*}}$ and $\hat{x}_{1}^{*}, \ldots, \hat{x}_{N}^{*} \in B_{X^{*}} \cap \operatorname{span} C$ such that

Notice that then

$$
\sqrt{\left\|x_{n}^{*}-\hat{x}_{n}^{*}\right\|}<\frac{\varepsilon}{4 M c}, \quad n=1, \ldots, N .
$$

$$
\left\|x_{n}^{*}-\hat{x}_{n}^{*}\right\|<\frac{\varepsilon}{4 M}, \quad n=1, \ldots, N .
$$

Let us denote by $K$ the closed absolutely convex hull in $X^{*}$ of the compact set $C \cup\left\{\hat{x}_{1}^{*}, \ldots, \hat{x}_{N}^{*}\right\} \cup\left\{0, x_{1}^{*}, x_{2}^{*}, \ldots\right\}$. Since $K \subset B_{X^{*}}$ and $K$ is compact, by the factorization lemma, there exists a reflexive Banach space $Y$, which is a linear subspace of $X^{*}$, such that the identity embedding $J: Y \rightarrow X^{*}$ is compact and $\|J\| \leq 1$. Moreover,

$$
C \cup\left\{\hat{x}_{1}^{*}, \ldots, \hat{x}_{N}^{*}\right\} \cup\left\{0, x_{1}^{*}, x_{2}^{*}, \ldots\right\} \subset J\left(B_{Y}\right) .
$$

Since $X$ has the weak MAP, for the operator $\left.J^{*}\right|_{X} \in \mathcal{K}\left(X, Y^{*}\right)$, there exists a net $\left(S_{\alpha}\right) \subset \mathcal{F}(X, X)$ with $\left\|\left.J^{*}\right|_{X} S_{\alpha}\right\| \leq 1$ for all $\alpha$ such that $J^{*} S_{\alpha} x \rightarrow$ $J^{*} x$ in $Y^{*}$ for all $x \in X$. This yields that $\left(S_{\alpha}^{*} J y\right)(x) \rightarrow(J y)(x)$ for all $y \in Y$ and $x \in X$. Observe that $\left\|S_{\alpha}^{*} J\right\| \leq 1$ for all $\alpha$ because $S_{\alpha}^{*} J=\left(\left.J^{*}\right|_{X} S_{\alpha}\right)^{*}$ and $\left\|\left.J^{*}\right|_{X} S_{\alpha}\right\| \leq 1$.

Consider now any $x^{*} \in C$. Then $x^{*}=J y$ with $y \in B_{Y}$, implying that $\left\|S_{\alpha}^{*} x^{*}\right\|=\left\|S_{\alpha}^{*} J y\right\| \leq\|y\| \leq 1$. Since $x^{*}$ is also a weak* strongly exposed point of $B_{X^{*}}$ and $\left(S_{\alpha}^{*} x^{*}\right)(x) \rightarrow x^{*}(x)$ for all $x \in X$, it follows that $S_{\alpha}^{*} x^{*} \rightarrow$ 
$x^{*}$ in $X^{*}$. Consequently, $S_{\alpha}^{*} x^{*} \rightarrow x^{*}$ for all $x^{*} \in \operatorname{span} C$, in particular, $S_{\alpha}^{*} \hat{x}_{n}^{*} \rightarrow \hat{x}_{n}^{*}$ for $n=1, \ldots, N$. Let us fix an $\alpha$ such that

$$
\left\|\hat{x}_{n}^{*}-S_{\alpha}^{*} \hat{x}_{n}^{*}\right\|<\frac{\varepsilon}{4 M}, \quad n=1, \ldots, N .
$$

Finally, recalling that $\hat{x}_{n}^{*}=J \hat{y}_{n}$ with $\hat{y}_{n} \in B_{Y}$ for $n=1, \ldots, N$, and $x_{n}^{*}=J y_{n}$ with $y_{n} \in B_{Y}$ for $n \in \mathbb{N}$, we have

$$
\begin{aligned}
& \left|\sum_{n=1}^{\infty} x_{n}^{* *}\left(x_{n}^{*}\right)\right| \\
= & \left|\sum_{n=1}^{\infty} x_{n}^{* *}\left(x_{n}^{*}\right)-\sum_{n=1}^{\infty} x_{n}^{* *}\left(S_{\alpha}^{*} x_{n}^{*}\right)\right| \\
\leq & \sum_{n=1}^{N}\left\|x_{n}^{* *}\right\|\left\|x_{n}^{*}-\hat{x}_{n}^{*}\right\|+\sum_{n=1}^{N}\left\|x_{n}^{* *}\right\|\left\|\hat{x}_{n}^{*}-S_{\alpha}^{*} \hat{x}_{n}^{*}\right\| \\
+ & \sum_{n=1}^{N}\left\|x_{n}^{* *}\right\|\left\|S_{\alpha}^{*} \hat{x}_{n}^{*}-S_{\alpha}^{*} x_{n}^{*}\right\|+\sum_{n>N}\left\|x_{n}^{* *}\right\|\left\|x_{n}^{*}-S_{\alpha}^{*} x_{n}^{*}\right\| \\
< & \frac{\varepsilon}{4}+\frac{\varepsilon}{4}+\sum_{n=1}^{N}\left\|x_{n}^{* *}\right\|\left\|\left(S_{\alpha}^{*} J\right)\left(\hat{y}_{n}-y_{n}\right)\right\|+\sum_{n>N}\left\|x_{n}^{* *}\right\|\left\|x_{n}^{*}-\left(S_{\alpha}^{*} J\right) y_{n}\right\| \\
\leq & \frac{\varepsilon}{2}+\sum_{n=1}^{N}\left\|x_{n}^{* *}\right\|\left\|\hat{y}_{n}-y_{n}\right\|+\sum_{n>N}\left\|x_{n}^{* *}\right\|\left(\left\|x_{n}^{*}\right\|+\left\|y_{n}\right\|\right) \\
< & \frac{\varepsilon}{2}+c \sum_{n=1}^{N}\left\|x_{n}^{* *}\right\| \sqrt{\left\|\hat{x}_{n}^{*}-x_{n}^{*}\right\|}+\frac{\varepsilon}{4} \\
< & \frac{3 \varepsilon}{4}+\frac{\varepsilon}{4}=\varepsilon .
\end{aligned}
$$

(Let us mention that the estimate

$$
\|y-z\|^{2} \leq c^{2}\|J y-J z\|, \quad y, z \in K,
$$

we used above, can be found in [13, page 335].) Hence, $\sum_{n} x_{n}^{* *}\left(x_{n}^{*}\right)=0$ as desired.

Remark 4.1. If $X^{*}$ has the Radon-Nikodým property, then $B_{X^{*}}=\overline{\operatorname{conv}} w^{*}\left(w^{*}\right.$-sexp $\left.B_{X^{*}}\right)$ (see, e.g., [18, page 86]). Hence $\overline{\operatorname{span}}\left(w^{*}\right.$-sexp $\left.B_{X^{*}}\right)$ is a norming subspace and must be equal to $X^{*}$ whenever $X^{*}$ contains no proper norming closed subspace. The condition $X^{*}=\overline{\operatorname{span}}\left(w^{*}-\operatorname{sexp} B_{X^{*}}\right)$ is satisfied if $X$ is a Banach space with property $\mathrm{M}^{*}(a, B, c), \max |B|+c>1$, introduced and studied in [15] and [16]. Any Banach space $X$ with separable dual can be equivalently renormed to have such a property (see [16, Proposition 1.2]). One has $X^{*}=\overline{\operatorname{span}}\left(w^{*}\right.$-sexp $\left.B_{X^{*}}\right)$ if $X$ is an M-ideal in $X^{* *}$ (see, e.g., [9, page 127]), in particular, if $X$ is a closed subspace of $c_{0}$.

Proof of Proposition 2.3. Let $X$ be the closed subspace of $c_{0}$ constructed by Johnson and Schechtman (see [11, Corollary JS]). Then $X$ has the 8-BAP (see $[6$, Theorem VI.3 and its proof $]$ ) and $X^{*}$ does not have the AP. As we mentioned in Remark 4.1, $X^{*}=\overline{\operatorname{span}}\left(w^{*}\right.$-sexp $\left.B_{X^{*}}\right)$. If $X$ had the weak 
MAP, then by Theorem 4.1, $X^{*}$ would also have the weak MAP, hence the AP. This is not the case.

We have seen that the weak MAP passes up from $X$ to $X^{*}$ whenever $X^{*}$ has the Radon-Nikodým property and $X^{*}$ contains no proper norming closed subspace. Godefroy and Saphar [7] have proved (in a completely different manner) that the MAP passes up from $X$ to $X^{*}$ whenever $X^{*}$ contains no proper norming closed subspace. Concerning the MAP, there is another well-known lifting result due to Johnson [10] (see, e.g., [1, page 289]): if $X$ has the MAP in every equivalent norm, then $X^{*}$ has the MAP. We show (the proof is completely different from Johnson's) that the same holds true for the weak MAP.

Theorem 4.2. Let $X$ be a Banach space. The following statements are equivalent.

(a) $X$ has the weak MAP in every equivalent norm.

(b) $X^{*}$ has the weak MAP.

(c) $X^{*}$ has the AP.

Proof. (a) $\Rightarrow$ (c). As in the proof of Theorem 4.1, we shall show that when $\left(x_{n}^{*}\right) \subset X^{*}$ and $\left(x_{n}^{* *}\right) \subset X^{* *}$ satisfy $\sum_{n=1}^{\infty}\left\|x_{n}^{*}\right\|\left\|x_{n}^{* *}\right\|<\infty$ and $\sum_{n=1}^{\infty} x_{n}^{* *}\left(S^{*} x_{n}^{*}\right)=$ 0 for all $S \in \mathcal{F}(X, X)$, then $\sum_{n=1}^{\infty} x_{n}^{* *}\left(x_{n}^{*}\right)=0$.

We may assume that $1 \geq\left\|x_{n}^{*}\right\| \rightarrow 0$ and

$$
M:=\sum_{n=1}^{\infty}\left\|x_{n}^{* *}\right\|<\infty .
$$

Let $\varepsilon>0$ and choose $N \in \mathbb{N}$ such that

$$
\sum_{n>N}\left\|x_{n}^{* *}\right\|<\frac{\varepsilon}{4}
$$

Let $F=\operatorname{span}\left\{x_{1}^{*}, \ldots, x_{N}^{*}\right\}$. Let $\left(\hat{X},\|\cdot\|_{\wedge}\right)$ be an equivalent renorming of $X$ such that $\hat{X}^{*}$ is locally uniformly rotund (LUR) on $F$. We can assume that $\left\|x^{*}\right\|_{\wedge} \leq\left\|x^{*}\right\|$ for all $x^{*} \in X^{*}$ (see, e.g., [12]). Let $K=\overline{\operatorname{absconv}}\left(B_{\hat{F}} \cup\right.$ $\left.\left(x_{n}^{*}\right)_{n=1}^{\infty}\right)$. Then $K \subset B_{\hat{X}^{*}}$ is compact. Using the factorization lemma, we find a reflexive Banach space $Z$, which is a linear subspace of $X^{*}$, such that the the identity embedding $J: Z \rightarrow \hat{X}^{*}$ is compact and $\|J\| \leq 1$. Moreover, $K \subset J\left(B_{Z}\right)$. Since $X$ has the weak MAP, for $T=\left.J^{*}\right|_{\hat{X}} \in \mathcal{K}\left(\hat{X}, Z^{*}\right)$, there exists a net $\left(S_{\alpha}\right) \subset \mathcal{F}(\hat{X}, \hat{X})$ such that $\sup _{\alpha}\left\|T S_{\alpha}\right\| \leq\|T\| \leq 1$ and $J^{*} S_{\alpha} x \rightarrow J^{*} x$ for all $x \in X$. For all $z \in Z$ and $x \in X$ we get $\left(S_{\alpha}^{*} J z\right)(x)=$ $\left(J^{*} S_{\alpha} x\right)(z) \rightarrow\left(J^{*} x\right)(z)=(J z)(x)$. Thus $S_{\alpha}^{*} x^{*} \rightarrow x^{*}$ weak $^{*}$ for all $x^{*} \in$ $J(Z)$. In particular, $S_{\alpha}^{*} x^{*} \rightarrow x^{*}$ weak* for all $x^{*} \in F$.

Since $\left\|T S_{\alpha}\right\| \leq 1$, we also have $\left\|S_{\alpha}^{*} J\right\| \leq 1$. If $x^{*} \in F$ and $\left\|x^{*}\right\|_{\wedge}=1$, then $x^{*}=J z$ for some $z \in B_{Z}$. Thus $\left\|S_{\alpha}^{*} x^{*}\right\|_{\wedge} \leq 1$. The norm of $\hat{X}^{*}$ is LUR on $\hat{F}$, so the weak* convergence $S_{\alpha}^{*} x^{*} \rightarrow x^{*}$ is also the $\|\cdot\|_{\wedge}$-norm convergence. Thus $S_{\alpha}^{*} x^{*} \rightarrow x^{*}$ also in the equivalent $\|\cdot\|$-norm. Hence, there is some $S_{\alpha}$ such that

$$
\left\|x_{n}^{*}-S_{\alpha}^{*} x_{n}^{*}\right\|<\frac{\varepsilon}{2 M}, \quad n=1, \ldots, N
$$


Now we get

$$
\begin{aligned}
\left|\sum_{n=1}^{\infty} x_{n}^{* *}\left(x_{n}^{*}\right)\right| & =\left|\sum_{n=1}^{\infty} x_{n}^{* *}\left(x_{n}^{*}-S_{\alpha}^{*} x_{n}^{*}\right)\right| \\
& \leq \sum_{n=1}^{N}\left\|x_{n}^{* *}\right\|\left\|x_{n}^{*}-S_{\alpha}^{*} x_{n}^{*}\right\|+\sum_{n>N}\left\|x_{n}^{* *}\right\|_{\wedge}\left\|J z_{n}-S_{\alpha}^{*} J z_{n}\right\|_{\wedge} \\
& \leq \frac{\varepsilon}{2}+\sum_{n>N}\left\|x_{n}^{* *}\right\|_{\wedge}\left(\|J\|+\left\|S_{\alpha}^{*} J\right\|\right)\left\|z_{n}\right\| \\
& \leq \frac{\varepsilon}{2}+\frac{\varepsilon}{2}=\varepsilon .
\end{aligned}
$$

Hence $\sum_{n=1}^{\infty} x_{n}^{* *}\left(x_{n}^{*}\right)=0$.

(c) $\Rightarrow$ (a). If $X^{*}$ has the AP, then $X^{*}$ has the AP in every equivalent norm. Therefore it suffices to prove that $X$ has the weak MAP. We are going to apply Theorem 3.2 (see also Remark 3.2). Let $Y$ be a reflexive Banach space and let $u \in X \otimes_{\pi} Y^{*}$. As in the beginning of the proof of Theorem 3.2, we find $T \in \mathcal{W}(X, Y)$ with $\|T\|=1$ such that $\|u\|_{\pi}=\operatorname{trace}(T u)$. By a criterion of the AP for $X^{*}$ in $\left[17\right.$, Theorem 5], there exists a net $\left(T_{\alpha}\right) \subset \mathcal{F}(X, Y)$ with $\sup _{\alpha}\left\|T_{\alpha}\right\| \leq\|T\|=1$ such that $T_{\alpha}^{*} y^{*} \rightarrow T^{*} y^{*}$ for all $y^{*} \in Y^{*}$. But then

$$
\begin{aligned}
\|u\|_{\pi} & =\operatorname{trace}(T u)=\lim _{\alpha} \operatorname{trace}\left(T_{\alpha} u\right) \\
& \leq \sup _{\alpha}\left|\operatorname{trace}\left(T_{\alpha} u\right)\right|=\sup _{\alpha}\left|(V u)\left(T_{\alpha}\right)\right| \leq\|V u\|
\end{aligned}
$$

as desired.

(b) $\Leftrightarrow(\mathrm{c})$. See Theorem 3.6.

It is clear from Theorem 4.2 that Problems 1.1 and 1.4 (see Introduction) are equivalent.

\section{REFERENCES}

[1] P.G. Casazza. Approximation properties. In: W.B. Johnson and J. Lindenstrauss (eds.) Handbook of the Geometry of Banach Spaces. Volume 1, Elsevier (2001) 271316 .

[2] W.J. Davis, T. Figiel, W.B. Johnson, and A. Peeczyński. Factoring weakly compact operators. J. Funct. Anal. 17 (1974) 311-327.

[3] A. Defant and K. Floret. Tensor Norms and Operator Ideals. North-Holland Mathematics Studies 176 (1993).

[4] J. Diestel And J.J. Uhl, JR. Vector Measures. Mathematical Surveys 15, Amer. Math. Soc., Providence, Rhode Island (1977).

[5] M. Feder And P.D. SAPhar. Spaces of compact operators and their dual spaces. Israel J. Math. 21 (1975) 38-49.

[6] G. Godefroy. The Banach space $c_{0}$. Extracta Math. 16 (2001) 1-25.

[7] G. Godefroy and P.D. Saphar. Duality in spaces of operators and smooth norms on Banach spaces. Illinois J. Math. 32 (1988) 672-695.

[8] A. Grothendieck. Produits tensoriels topologiques et espaces nucléaires. Mem. Amer. Math. Soc. 16 (1955).

[9] P. Harmand, D. Werner, And W. Werner. M-ideals in Banach Spaces and Banach Algebras. Lecture Notes in Math. 1547, Springer-Verlag, Berlin-Heidelberg (1993).

[10] W.B. Johnson. A complementary universal conjugate Banach space and its relation to the approximation problem, Israel J. Math. 13 (1972), 301-310. 
[11] W.B. Johnson And T. OikhBerG. Separable lifting property and extensions of local reflexivity. Illinois J. Math. 45 (2001) 123-137.

[12] V. Lima AND A. Lima. Ideals of operators and the metric approximation property. J. Funct. Anal. 210 (2004) 148-170.

[13] Å. Lima, O. NygaARd, AND E. OJA. Isometric factorization of weakly compact operators and the approximation property. Israel J. Math. 119 (2000) 325-348.

[14] J. Lindenstrauss and L. Tzafriri. Classical Banach Spaces I. Ergebnisse der Mathematik und ihrer Grenzgebiete 92, Springer-Verlag (1977).

[15] E. OJA. Géométrie des espaces de Banach ayant des approximations de l'identité contractantes. C. R. Acad. Sci. Paris, Sér. I, 328 (1999) 1167-1170.

[16] E. OJA. Geometry of Banach spaces having shrinking approximations of the identity. Trans. Amer. Math. Soc. 352 (2000) 2801-2823.

[17] E. Oja AND A. Pelander. The approximation property in terms of the approximability of weak ${ }^{*}$-weak continuous operators. J. Math. Anal. Appl. 286 (2003) 713-723.

[18] R. Phelps. Convex Functions, Monotone Operators and Differentiability. Second edition. Lecture Notes in Math. 1364, Springer-Verlag (1993).

[19] O.I. Reinov. Un contre-exemple à une conjecture de A. Grothendieck. C. R. Acad. Sc. Paris, Sér. I, 296 (1983) 597-599.

[20] R.A. RYAN. Introduction to Tensor Products of Banach Spaces. Springer Monographs in Mathematics, Springer-Verlag (2002).

Department of Mathematics, Agder University College, Serviceboks 422, 4604 Kristiansand, NorWAY

E-mail address: Asvald.Lima@hia.no

Faculty of Mathematics and Computer Science, Tartu University, J. Litvi

2, EE-50409 TARTU, Estonia

E-mail address: eve.oja@ut.ee 